\title{
Accountability for the Quality of Care Provided to People with Serious IIIness
}

\author{
Maureen Henry, JD, PhD, Sarah Hudson Scholle, MPH, DrPH, and Jessica Briefer French, MHSA
}

\section{Abstract}

Background: Care for patients with serious illness is an emerging practice area that has gained attention as valuebased purchasing has increased. While the number of programs is growing, their impact on care quality and outcomes is unknown.

Objective: With support from the Gordon and Betty Moore Foundation, the National Committee for Quality Assurance (NCQA) is assessing the feasibility of creating an accountability program focused on serious illness care.

Methods: This article describes the process of developing an accountability program, findings from our initial work, and our plans to develop measures for a serious illness care accountability program. We focused on three questions:

1. What patient populations should be targeted for measurement?

2. What entities have accountability for ensuring high-quality care for serious illness?

3. What structures, processes, and outcomes should be evaluated in an accountability program for serious illness care?

Results: Our environmental scan showed that the evidence base for specific patient populations or care models is not sufficiently mature to justify traditional structure and process measures. In visits to serious illness care programs, we observed different staffing models, care models, care settings, and payment structures. We found a gap between recommended inclusion criteria and services when compared to inclusion criteria and services offered by existing programs.

Conclusions: To address the challenges, NCQA intends to develop outcome measures driven by patient and family priorities. Structure and process measures will focus on building organizations' capacity to measure outcomes, including patient engagement and outcomes, linked to patient goals.

Keywords: accountability programs; quality of care; measure development; serious illness; value-based purchasing

\section{Purpose of the Article}

$\mathbf{T}$ HE NUMBER OF AMERICANS with serious illness is rising, along with overall costs of care. ${ }^{1}$ Patients with serious illness too often receive expensive care that is misaligned with their goals, values, and preferences. ${ }^{1}$ The current shift away from traditional fee-for-service payment to value-based purchasing presents opportunities to improve care and goal alignment, but it also comes with the risk that pressures to lower costs will reduce quality. Ideally, value-based purchasing will direct resources toward high-quality care that aligns with patients' goals, values, and preferences.

Serious illness care programs have arisen to meet the care needs of seriously ill patients and their families. These programs are heterogeneous, with diverse definitions of serious illness ${ }^{2-4}$ and serious illness care ${ }^{2,3,5}$ and

National Committee for Quality Assurance (NCQA), Washington, DC.

Accepted November 3, 2017.

This article was developed for, and discussed at, a meeting titled "A Convening on Quality Measures for Serious Illness Care," which was held May 9-11, 2017 in Banff, Canada. The meeting was organized by the Gordon and Betty Moore Foundation, the Cambia Palliative Care Center of Excellence at the University of Washington, the Center to Advance Palliative Care, and the Icahn School of Medicine at Mount Sinai. This supplement is funded by the Gordon and Betty Moore Foundation. 
an array of care models, program staffing, settings, and organizational sponsors. ${ }^{2,5}$ But a dearth of performance measures and accountability programs addressing the quality of such care means that consumers lack the means to identify health plans, serious illness care programs, or providers delivering high-quality person- and familycentered care. ${ }^{2}$

With support from the Gordon and Betty Moore Foundation, the National Committee for Quality Assurance (NCQA) is developing serious illness care performance measures and evaluating the potential for serious illness care accountability programs.

This article describes NCQA's process for developing an accountability program, findings from an environmental scan, and plans to develop measures suitable for a serious illness care accountability program.

\section{Developing a Serious IIIness Care Accountability Program}

One goal of this project is to evaluate the feasibility of developing accountability programs for serious illness care at the provider, program, Accountable Care Organization (ACO), and plan levels of accountability. To evaluate the potential for accountability programs, we will draw on NCQA's established processes for program and measure development. Initial efforts will address three crucial questions that guide development:

1. What patient populations should be targeted for measurement?

2. What entities have accountability for ensuring highquality patient care for serious illness?

3. What structures, processes, and outcomes should be evaluated in an accountability program for serious illness care?

\section{Accountability programs}

Accountability program development includes a feasibility review, product design, and implementation infrastructure design (Fig. 1). The feasibility review defines the product concept and then evaluates current gaps and the market for potential customers. Product design includes cyclical development and refinement of the product concept and requirements (including measure development), testing and public comment, and internal reviews. Infrastructure design includes data collection tools, program application, supporting materials, and the product release plan. Throughout this process, NCQA engages multistakeholder panels to obtain input and seeks public comment on proposed program components.

\section{Measures for accountability}

Measure development (Fig. 2) is critical in accountability program design. To develop a measure, NCQA measure developers define measure concepts that address important quality gaps (identified through an environmental scan and key informant interviews), draft measure specifications, and test draft measures. A broad set of external stakeholders, such as patients and family caregivers, providers, purchasers, plans, and researchers, inform the measure development process.

\section{Approach}

To understand the evidence for serious illness care practices, we conducted an environmental scan focused on guidelines, existing measures, and recommendations made by national organizations. We also conducted site visits with 14 serious illness care programs that agreed to serve in a learning collaborative to support and inform program and measure development. During site visits, we learned about programs' target patient populations and inclusion criteria, care models, sponsorship, and financing structures, which helped us answer the key questions.

\section{Environmental scan}

The environmental scan targeted existing guidelines, recommendations, and measures, which we mapped against National Consensus Project for Quality Palliative Care (NCP) Guideline domains. ${ }^{6}$ We mapped recommendations from the Institute of Medicine Committee on Approaching Death: Addressing Key End-of-Life Issues, ${ }^{1}$ the Coalition to Transform Advanced Care, ${ }^{7}$ the National Quality

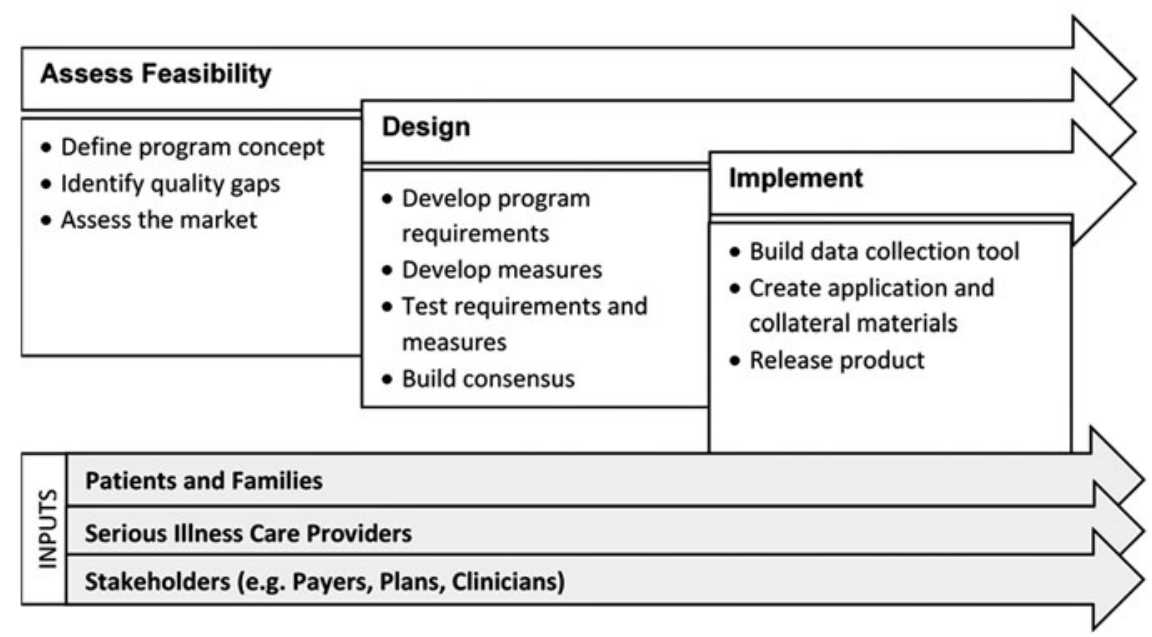

FIG. 1. NCQA accountability product development process. NCQA, National Committee for Quality Assurance. 


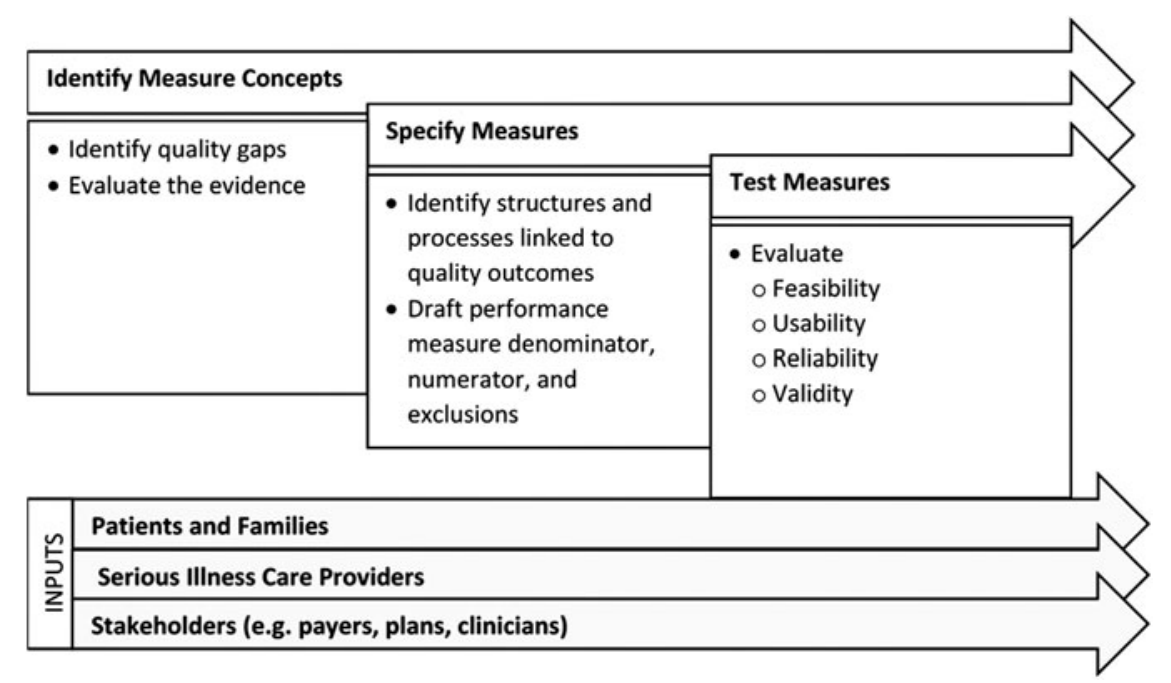

FIG. 2. NCQA measure development.

Forum, ${ }^{8}$ and the Center to Advance Palliative Care. ${ }^{9}$ We also mapped the PEACE, ${ }^{10,11}$ Assessing Care of Vulnerable Elders, ${ }^{12}$ and the Australian Palliative Care Outcomes Collaboration ${ }^{13}$ measure sets.

We weighed the relevance of the Center for Medicare and Medicaid Services (CMS) reporting requirements for organizations providing community-based serious illness care, including hospices, home health agencies, and ACOs, and NCQA standards addressing home-based care delivery (Accreditation of Case Management for Long-Term Services and Supports [LTSS] and LTSS Distinction for Health Plans) and oncology (Oncology Medical Home Recognition). Last, we identified systematic literature reviews and results of recent studies on community-based serious illness care to evaluate the evidence base for measurement.

\section{Learning collaborative site visits}

Involving serious illness care programs in measure development will help the team evaluate measure feasibility, reliability, and validity across different program models. To emphasize the voice of patients and caregivers in this project, we contracted with two paid patient advisors to serve as team members and recruited two patients and one family caregiver to serve on the stakeholder panel. We also required each learning collaborative site to convene a patient and family advisory panel with at least six members who either live with serious illness or are family caregivers of patients with serious illness (66 individuals, total); these panels will provide local input on serious illness care measures and quality improvement efforts.

The learning collaborative comprises 11 serious illness care programs in 9 states: Alabama, California, Illinois, New Jersey, New York, North Carolina, Pennsylvania (2), Utah (2), and Virginia. Organizational homes for the programs include hospice (1), Veterans' Administration Medical Center (1), academic medical center (1), stand-alone hospice agency (1), medical groups (2), and health systems (5). Ten programs are within larger nonprofit organizations. While most have a broad population focus, two programs provide specialty care (oncology and pulmonology) and two provide primary care.
In recruiting learning collaborative sites, we sought diversity in organizational type, population served, and care models. All programs that we considered had been identified as exemplary 9,14 or had program elements or structures not otherwise represented among the other sites under consideration for inclusion. Programs were required to provide ambulatory or home-based serious illness care to 100 or more patients per month to be considered. We conducted in-person site visits (with the exception of two that were telephonic) lasting between four and six hours to evaluate whether programs met project inclusion criteria. Between 3 and 10 staff members participated from each program or parent organization. Site visits addressed serious illness decision making, care planning and documentation, disease and symptom management, emotional/psychological/social/spiritual care, and caregiver support. For each topic, program staff described their approach to care delivery, ongoing quality measurement and improvement activities, training, and health information technology. We also asked about current participation in demonstration or accountability programs.

\section{Findings \\ What patient population should be targeted for serious illness care measurement?}

The environmental scan suggested that no single narrative or operational definition of serious illness was used consistently across serious illness care programs. ${ }^{1,4}$ Serious illness was defined by one or more of the following elements: life expectancy, limited treatment options, decline in functional status, decline in quality of life, specific chronic conditions, multiple chronic conditions, high symptom burden, and/or high utilization.

The NCP Clinical Practice Guidelines for Quality Palliative Care, 4th edition project ${ }^{15}$ has adopted Kelley's definition of serious illness: "a health condition that carries a high risk of mortality and either negatively impacts a person's daily function or quality of life or excessively strains their caregiver.",

Inclusion criteria for enrolling in a serious illness program were diverse across programs we visited, but all used one or 
more elements listed above. Although most described their services as being "upstream from hospice," most served a more narrowly defined population than would be included in Kelley's definition ${ }^{4}$; most programs reported a mean length of stay of between six months and one year. The gap between proposed inclusive definitions, such as Kelley's, and the reality of restrictive inclusion criteria in serious illness care programs will pose a challenge for measure development.

Population definitions that serve as measure denominators have different implications for different levels of accountability. For serious illness program-level measurement, all enrolled patients can be included in measures because all patients, by definition, have serious illness.

In contrast, for population-based measurement of ACOs and health plans, only seriously ill patients should be included in the denominator when measures encourage specific interventions designed to benefit seriously ill patients, but of less or no value to patients without serious illness. Measure denominators must strike a balance between specificity and sensitivity. A narrow, highly specific denominator can be advantageous because it is less likely to encourage unnecessary care or services for patients who are unlikely to benefit—-but a measure that is too specific may limit measurement to only a small subset of patients who would benefit from specialized care. A broad, highly sensitive denominator will capture most patients with serious illness, along with many patients without serious illness.

\section{What entity should be accountable for serious illness care?}

Accountability programs and measures apply to precisely defined entities in healthcare delivery. Entities involved in serious illness care include health plans, ACOs, medical groups, home health agencies, hospices, and specialty organizations operating under medical provider licenses. ${ }^{1,7,9}$

When developing accountability programs and specifying performance measures, it is critical to match program requirements to actions within the measured entity's control. Measures for clinicians, groups, and programs can focus on assessment, care planning, care coordination, and care delivery. A medical practice delivering serious illness care may be required to send a comprehensive care plan to a hospital when admitting a patient, but would generally lack the ability to influence a hospital's actions. Performance measurement at the program or provider level is ill-suited to achieve systemic change across silos.

In contrast, measurement at the plan or ACO level can work across silos and address care coordination, care outcomes, and utilization. Clinician and group measures can also be adapted for use at the plan or ACO level to improve care for all seriously ill patients within the population, not just those who are fortunate enough to enroll in a serious illness care program.

It is important to consider how the number of patients included in a measure differs by type of organization. If the number is too low, it becomes difficult to measure performance reliably. Program-level measurement risks this challenge, but even measures that may not reliably distinguish between different programs have the potential to inform health plans or other payers considering creation of a serious illness care benefit or contracting with serious illness care programs.

\section{What structures and processes should organizations be accountable for when delivering serious illness care?}

Serious illness care models are designed to address care and support needs of seriously ill patients and their families. Models range from the well-defined and established (e.g., hospice and in-patient palliative care) to emerging care models (e.g., out-patient palliative care clinics and homebased primary care for home-bound elderly). While studies demonstrating positive utilization outcomes and cost savings associated with palliative care are promising, the evidence base for quality-of-life outcomes, improved symptom management, and the specific program elements is weak.

A recent meta-analysis of patient and family outcomes associated with palliative care found associations between palliative care and improvements in advanced care planning, patient and caregiver satisfaction, and lower healthcare utilization, but associations with caregiver and symptom burden outcomes were mixed. ${ }^{16}$ In a systematic review of randomized trials of early outpatient and home-based palliative care by Davis et al., ${ }^{17}$ study findings were inconsistent. Some showed improved quality of life and reduced cost of care; others showed no improvement in quality of life and no difference in cost of care compared with usual care. The authors attributed differences to methodological challenges, in part, including heterogeneous care models and patient populations. ${ }^{17}$

More recent studies have shown financial benefits from community-based serious illness care. Lustbader et al. ${ }^{18}$ found that home-based palliative care provided to patients in an ACO was associated with cost savings, reduced hospitalization, and increased hospice use. Another study compared the impact of three community-based oncology care programs that used different models to reduce cost and improve quality of life. ${ }^{19}$ All three models were associated with decreased costs of care in the last 30,90, and 180 days of life. One model, which used lay navigators, was associated with decreased hospitalization and emergency department visits and increased hospice use.

There are no evidence-based guidelines for communitybased palliative care. The NCP's consensus-based guidelines for palliative care were developed for hospice and hospitalbased palliative care programs. ${ }^{6}$ The NCP is updating the guidelines to address components of care-beyond those focused on hospital-based care- that are necessary for homebased serious illness care, such as patient-safety guidelines for providers in a patient's home. ${ }^{15}$ Recent expert consensus recommends that patients with serious illness have access to a broad range of program elements, from 24/7 telephone support to home-based care from a palliative physician. ${ }^{1}$

Our site visits showed that even among the group of programs identified as high performing, most programs do not offer the recommended comprehensive scope of services. We observed program elements that spanned from low-touch telephonic case management to high-touch, home-based palliative and primary care (Fig. 3). Some programs provided 24/7 telephone support, using nurses who were not trained in palliative care, to address the needs of high-utilizing patients. Some programs emphasized primary palliative care. In one program, nurses conducted home visits for assessment, but not care delivery. On the other end of the spectrum, some programs provided in-home medical, nursing, and/or social 


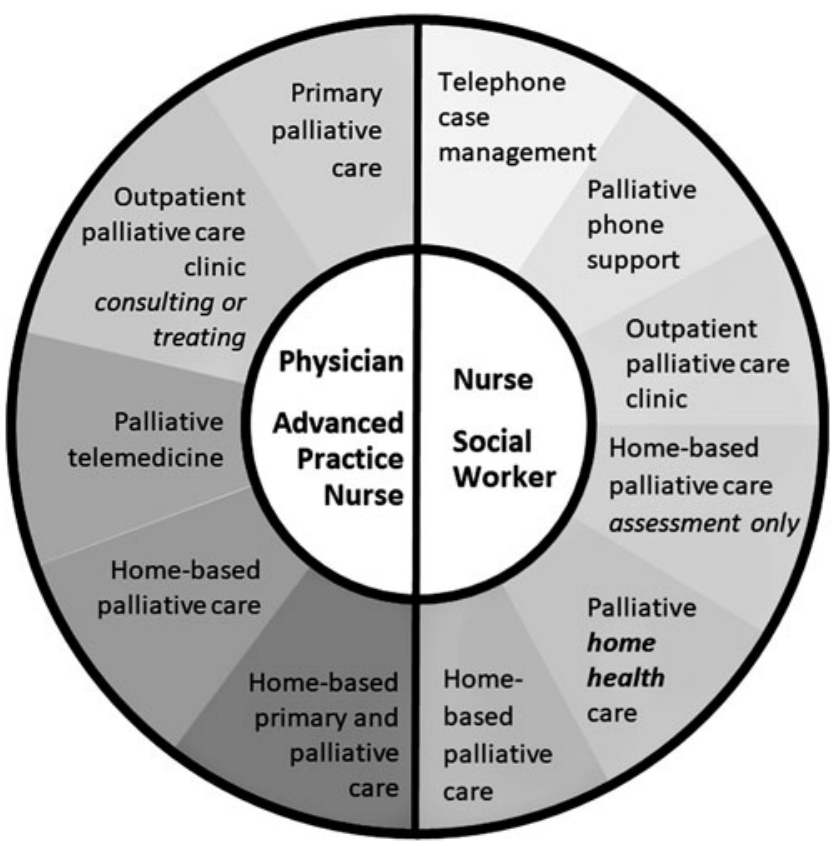

FIG. 3. Serious illness care program characteristics.

work care addressing palliative, behavioral health, and primary care needs.

Staffing models also differed among programs. Some expansive programs had interdisciplinary teams that included one or more physicians, an advanced practice registered nurse (APRN), registered nurse and/or licensed practical nurse, social worker, pharmacist, and chaplain. Other programs had smaller teams, such as an APRN/social work home-visit team supported by consultant physicians and a pharmacist, or a physician/nurse/social worker model.

All programs used an assessment and care plan, but specific elements addressed differed. Similarly, although each program engaged in advanced care planning and conversations about serious illness, most of them either did not follow a structured protocol (e.g., Vital Talk, Serious Illness Conversations, or Respecting Choices) and relied instead on clinician training and experience or combined elements of different structured protocols.

When creating accountability programs or measures that require a program to offer specific care or services or to use specific staffing models or deliver care in specific settings, a link between the required structures or processes and beneficial patient outcomes is critical. The strongest link is a robust evidence base, ideally in the form of evidence-based practice guidelines. Consensus guidelines can be used to make the link, but they are weaker than evidence-based guidelines. Expert opinion can be used to support inclusion of specific care elements, structures, or processes in an accountability program, but it provides the weakest link between the care element and beneficial outcomes. Diversity of care models, services, and staffing points to a lack of consensus about what a serious illness program should offer.

\section{Discussion}

To successfully develop measures for serious illness accountability programs, NCQA must have a strategy for de- fining the target population, determining the right levels of accountability, and navigating an environment with a developing evidence base and a gap between expert recommendations and services offered.

As we continue with the project, we will consider the evidence linking improved outcomes to structures and processes when applied to a precisely defined population of seriously ill patients. We will also evaluate the degree to which the definition should promote access to serious illness care to a broad patient population, when most organizations currently lack the financial and workforce resources needed to meet aspirational standards.

We have concluded that it is necessary to target both provider groups/programs and plans/ACOs for accountability and measurement, to fill gaps in clinical care performance measurement and in meeting the needs of people with serious illness at the population level.

The lack of evidence for specific elements of communitybased serious illness care and the heterogeneity among care models make it premature to prescribe specific care or services, staffing models, or site of care in an accountability program. Given the immaturity of the evidence, we will focus on process measures that build the infrastructure needed for outcome measures addressing symptoms, functioning, engagement, and experience with care. We are working toward measurement approaches that consider patients' priorities for outcomes and goals.

Accountability program and measure development will emphasize outcomes that are important to patients and families, data needed to measure those outcomes, and standards and processes needed to capture the data needed for outcome measures, as well as any measures that are critical to patient protection that may not be adequately addressed in outcome measures. This approach avoids the pitfalls of prematurely aligning measures with emerging care models and instead allows organizations to use innovation to find the best path to high-quality serious illness care.

\section{Acknowledgment}

Supported by the Gordon and Betty Moore Foundation.

\section{Author Disclosure Statement}

No competing financial interests exist.

\section{References}

1. Institute of Medicine: Dying in America: Improving the Quality and Honoring Individual Preferences Near the End of Life. Washington, DC: National Academies Press, 2014.

2. Cohn J, Corrigan J, Lynn J, et al.: Community-Based Models of Care Delivery for People with Serious Illness. Washington, DC: National Academies of Medicine, 2017.

3. National Coalition for Hospice and Palliative Care: $\mathrm{Na}$ tional Consensus Project Stakeholder Strategic Directions Summit: Summary Report. Chicago, IL: National Coalition for Hospice and Palliative Care, 2017.

4. Kelley AS: Identifying the population with serious illness: The "denominator" challenge. J Palliat Med 2018;S2: S7-S16.

5. Coalition to Transform Advanced Care (C-TAC): Toward a Serious Illness Program Design and Implementation Framework. Washington, DC: C-TAC, 2017. 
6. National Consensus Project for Quality Palliative Care (NCP): Clinical Practice Guidelines for Quality Palliative Care, 3rd ed. Pittsburgh, PA: NCP, 2013.

7. C-TAC: Policy agenda: Options to transform advanced care. www.thectac.org/wp-content/uploads/2015/02/C_TACPolicy-Agenda.pdf. (Last accessed April 4, 2017).

8. National Quality Forum: 2016 palliative and end-of-life care, 2015-2016 technical report. www.qualityforum.org/ Publications/2016/12/Palliative_and_End-of-Life_Care_20152016.aspx. (Last accessed April 10, 2017).

9. Center to Advance Palliative Care: Palliative Care in the Home: A Guide to Program Design. New York: Center to Advance Palliative Care, 2017.

10. Schenck AP, Rokoske FS, Durham DD, et al.: The PEACE Project: Identification of quality measures for hospice and palliative care. J Palliat Med 2010;13:1451-1459.

11. PEACE Hospice and Palliative Care Quality Measures: www .med.unc.edu/pcare/resources/PEACE-Quality-Measures. (Last accessed October 3, 2017).

12. RAND: Assessing Care of Vulnerable Elders (ACOVE). www.rand.org/health/projects/acove.html. (Last accessed October 3, 2017).

13. Palliative Care Outcomes Collaboration (PCOC): http://ahsri .uow.edu.au/pcoc/about/index.html. (Last accessed October 3, 2017).

14. Kerr K: Community-Based Model Programs for the Seriously Ill. Palo Alto, CA: Gordon and Betty Moore Foundation, 2017.
15. National Consensus Project for Quality Serious Illness Care: 4th Edition Project Overview and Scope: www .nationalcoalitionhpc.org/wp-content/uploads/2017/04/NCPOverview-and-Scope-8.23.17-1.pdf. (Last accessed October 3, 2017).

16. Kavalieratos D, Corbelli J, Zhang D, et al.: Association between palliative care and patient and caregiver outcomes: A systematic review and meta-analysis. JAMA 2016;316: 2104-2114.

17. Davis MP, Temel JS, Balboni T, et al:: A review of the trials which examine early integration of outpatient and home palliative care for patients with serious illnesses. Ann Palliat Med 2015;4:99-121.

18. Lustbader D, Mudra M, Romano C, et al.: The impact of a home-based palliative care program in an Accountable Care Organization. J Palliat Med 2017;20:23-28.

19. Colligan EM, Ewald E, Ruiz S, et al.: Innovative oncology care models improve end-of-life quality, reduce utilization and spending. Health Affairs 2017;36:433440 .

Address correspondence to:

Maureen Henry, JD, PhD

National Committee for Quality Assurance (NCQA) 1100 13th Street NW, Third Floor Washington, DC 20005

E-mail: henry@ncqa.org 